\title{
Intermittent hypoxia training effects on rat skeletal muscle SDH
}

\author{
Qiaozhen Yan, Fangtao Liu and Jianfu Zhu \\ School of Physical Education, Wenshan University, Wenshan 663099, China \\ qiaozhen@163.com
}

Keywords: intermittent hypoxia training, skeletal muscle, succinodehydrogenas

\begin{abstract}
Based on 72 SD male rats were intermittent hypoxia training, observe its quiet state three hours after, immediately after exercise, and exercise changes the activity of SDH in skeletal muscle. Found that in the condition of the three, the rat skeletal muscle SDH has obvious changes, and presents the downward trend after rising first, including hypoxia plus exercise group of the most significant change.
\end{abstract}

\section{Introduction}

Currently, hypoxic training on aerobic capacity research is one of the characteristics of domestic and foreign scholars research, but low oxygen training in rat skeletal muscle aerobic metabolism enzyme research is uncommon. This experiment intermittent hypoxia training model is established, the SD male rats for 4 weeks of intermittent hypoxia training, observation of rat skeletal muscle succinate dehydrogenase in quiet state, immediately after exercise, and the change of three hours after the movement, as a reference of hypoxic training on science.

\section{The research contents and methods}

The object of study.72 SD male rats, 8 weeks, according to the experimental animal feeding norm cage, adapt to the training 1 week after the experimental animals were randomly divided into 4 groups, formal training for three weeks, a total of 4 weeks.

\section{Experiment method.}

Experimental group. To adapt to the training of rats were randomly divided into 4 groups: a week after the often oxygen control group (C), often aerobic exercise group (S), intermittent hypoxic control group (I), intermittent hypoxic training group (IS), and each group of 18.

\section{Group training arrangement.}

(1)The control group (C): often oxygen normal food, free drinking water without any training activities.

(2)Often aerobic exercise group (S): rats only aerobic endurance training every day.

(3) The intermittent hypoxia group (I): the hypoxia group in low oxygen chamber IHT, three times a week, 1 hour each day (a combination of low oxygen for 5 minutes, 5 minutes intermittent, a total of six combinations).Oxygen concentration was $14 \%$, the first week after week, weekly decline $1 \%$.

(4) The intermittent hypoxia training group (IS) : hypoxia group in low oxygen chamber IHT, three times a week, 1 hour each day (a combination of low oxygen for 5 minutes, 5 minutes intermittent, a total of six combination).Oxygen concentration was $14 \%$, the first week after week, weekly decline $1 \%$, immediately after hypoxic training for aerobic endurance training every day. 
Movement patterns: sports group in domestic rats electric treadmill training, match the pace of week $15 \mathrm{~m} / \mathrm{min}$, $30 \mathrm{~min}$ every day, make animals familiar treadmill exercise, start increasing weekly load after 1 week, weekly increase the speed of $5 \mathrm{~m} / \mathrm{min}, 10 \mathrm{~min}$ increase time; The last week of speed up to $30 \mathrm{~m} / \mathrm{min}$, the time for $60 \mathrm{~min}$.

\section{The experimental materials}

After the training program, with $0.4 \%$ pentobarbital sodium LML/100 g weight to anesthesia in rats, quickly remove the calf muscles and heart, the heart on the apparently being weighed, and then use eye small cut separate left and right ventricle, left ventricular wall thickness is measured with vernier caliper, gastronomies muscle and myocardial put into $-70^{\circ} \mathrm{C}$ refrigerator.

\section{Test index}

Succinate dehydrogenase (SDH): according to the Nanjing built succinate dehydrogenase (SDH) checkerboard manual testing

The steps of the test indicators. Accurate said myocardial, posterior calf muscles of each $200 \mathrm{mg}$, respectively in small beaker $5 \mathrm{ml}$; With liquid move in advance in the refrigerator $\left(4^{\circ} \mathrm{C}\right)$ precooling of $1 \mathrm{ml}$ 9\% saline water in the beaker, eye small scissors cut up as soon as possible organization block (small beaker into ice water).Pour into a glass with a cut up tissue homogenate in the tube, then take $0.8 \mathrm{ml}$ cold saline flushing residual tissue fragments in the beaker, homogenate in homogenate tube together, make organization fully homogenate, made from $10 \%$ homogenate. Preparation of good homogenate in refrigerated centrifuge $3000 \mathrm{r} / \mathrm{min}$, the centrifugal $10 \mathrm{~min}$, take supernatant, according to the determination of succinate dehydrogenase kit succinate dehydrogenase activity.

\section{Data processing}

All the data using the mean and standard deviation (X+/-SD), statistical processing with single factor analysis of variance method, all data are calculated on a computer using statistical software SPSS and, significant difference of $\mathrm{P}<0.05$.

\section{The experimental results}

We can be seen from table 1 succinate dehydrogenase in the tissues of the skeletal muscle changes: intermittent hypoxia mode: quiet state of intermittent hypoxia training group and often oxygen control, often aerobic exercise group, intermittent hypoxic control group all has very significant difference $(\mathrm{P}<0.01)$, intermittent hypoxic control group, no significant difference; Immediately after exercise state of intermittent hypoxia and often oxygen control, often oxygen movement control group had significant difference $(\mathrm{P}<0.05)$, intermittent hypoxic training group and often oxygen control, often aerobic exercise group were very significant differences $(\mathrm{P}<0.01)$; Three hours after the movement state of intermittent hypoxic control group and the oxygen control often has a very significant difference $(\mathrm{P}<0.01)$, intermittent hypoxic control group and aerobic exercise group had significant difference $(\mathrm{P}<0.05)$, intermittent hypoxic training group often oxygen control, often aerobic exercise group were very significant differences $(\mathrm{P}<0.01)$. 
Table 1: two kinds of low oxygen pattern effect on rat skeletal muscle of succinate dehydrogenase Units: U/mgprot

\begin{tabular}{|c|c|c|c|}
\hline grouping & resting state & $\begin{array}{c}\text { Immediately after a } \\
\text { workout }\end{array}$ & $\begin{array}{c}\text { Three hours after } \\
\text { exercise }\end{array}$ \\
\hline Normoxia & $2.62 \pm 0.23$ & $2.96 \pm 0.88$ & $2.20 \pm 0.89$ \\
\hline Aerobic exercise group often & $2.85 \pm 0.08$ & $2.42+1.08$ & $3.18+0.66$ \\
\hline Intermittent hypoxic control group & $3.17 \pm 0.45$ & 4.69+0.94 & $4.45 \pm 0.50^{\# \# @ ~}$ \\
\hline Intermittent hypoxia training group & $4.08 \pm 0.76^{\# \# @ \Delta \Delta}$ & $5.13+0.69^{\# \# @ @ ~}$ & 4.71+1.19\#\#@@ \\
\hline
\end{tabular}

Note:

(1)And oxygen often compare \# in the control group $(\mathrm{P}<0.05$, \# \# $\mathrm{P}<0.01$;

(2) Compared with aerobic exercise group often @ in P $<0.05$, @ @ P $<0.01$;

(3) Compared with intermittent hypoxic control group delta in $\mathrm{P}<0.05$, the delta delta in $\mathrm{P}<$ 0.01 ;

(4) Compared with intermittent hypoxia training group * in $\mathrm{P}<0.05$, ** $\mathrm{P}<0.01$;

\section{Analysis and discussion}

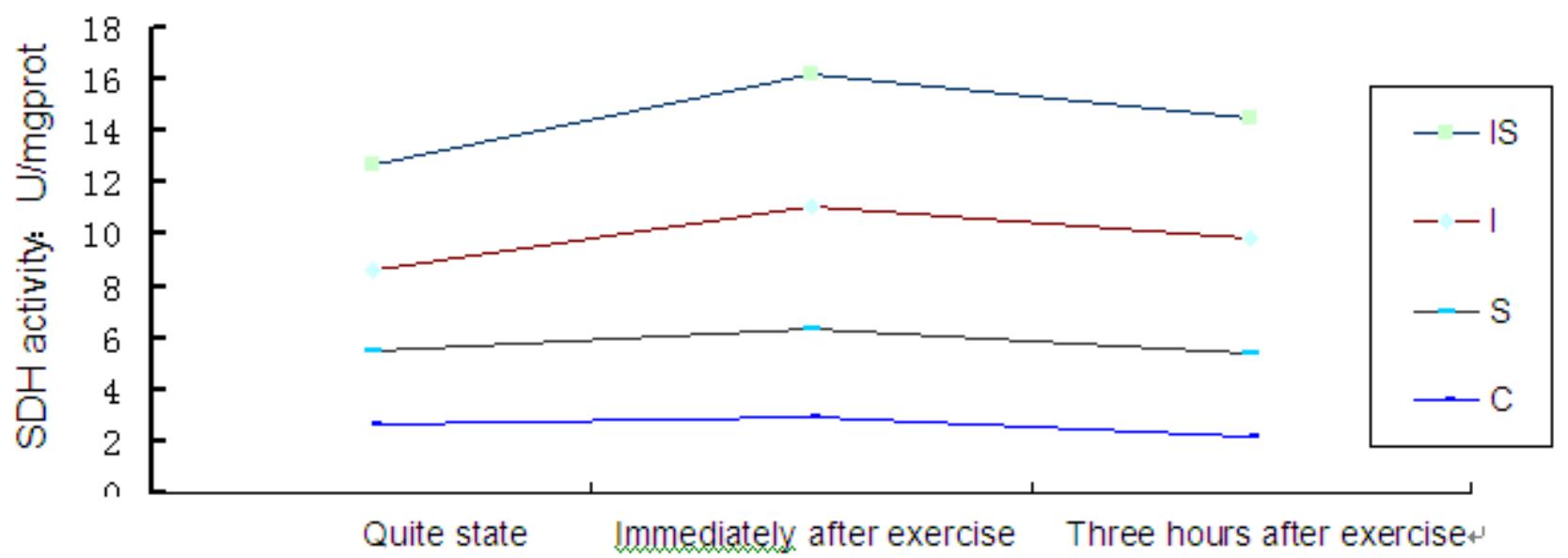

Figure 1: intermittent hypoxia training effects on rat skeletal muscle SDH

Succinatedehydrogenase(SDH) is the only mitochondrial membrane incorporation of three carboxylic acid cycle enzyme and is one of the most important reflect enzyme catalyzed aerobic oxidation ability of the body. SDH is often used to evaluate the effects of exercise training on aerobic capacity. SDH is a key enzyme in tricarboxylic acid cycle number three, level and content of its activity affects three carboxylic acid cycle speed. Three carboxylic acid cycle is the most important energy producing pathway during prolonged exercise of the body.

Lei Zhiping thinks that the intermittent hypoxic training can help to improve the brain, myocardium, skeletal muscle cytochrome oxidase (CCO), succinate dehydrogenase (SDH) activity in order to improve the organization oxygen utilization efficiency, thus effectively improving the overall aerobic metabolism ability. At the same time research shows, glycolytic enzyme mRNA's concentration were correlated with the intensity of training the and no obvious correlation with the effect of hypoxia. Desplanches found that intermittent hypoxia training, ratio of human muscle capillaries and muscle fibers increased 26\%. The number of mitochondria, capillary density and volume, citrate synthase activity increased significantly. GreenH research and training in intermittent hypoxia environment (13.5\% oxygen, 3 times a week, for 8 weeks) had found that citrate synthase activity increased 70\%[1].NemirovskayaT.L intermittent hypoxic training on 
rat.The simulated altitude of 5000 meters, 18 hours and 2 hours a day and totally 2 weeks,the skeletal muscle capillary density did not change obviously. The domestic and foreign research on skeletal muscle metabolic enzymes reported intermittent hypoxic training rats myocardium, skeletal muscle aerobic metabolic enzymes ( $\mathrm{MDH}[2]$, SDH and $\mathrm{CCO}[3]$ ) increased significantly, movement time prolonged. The increasing load of intermittent hypoxic exercising rats myocardial ATPase activity obviously increased, the swimming time was significantly prolonged [4].From this we can see that reasonable intermittent hypoxic training can make the muscle cells under appropriate stimulation and make muscle structure and function of cells produce adaptive changes,so that increased muscle cell enzyme activity, improve the organization using the oxygen ability. reasonable intermittent hypoxic training can also help conducive to cardiac and skeletal muscle aerobic metabolism and ensure energy supply to muscle tissue collected lapse, improve muscle work efficiency and a lasting movement ability. Normoxic training combined with double stimulation of hypoxia, conducive to the recruitment of slow twitch motor units in gastrocnemius muscle containing oxidase activity is high,thereby promoting the aerobic oxidation ability improvement.Studies have shown that differences in exercise training (mainly the strength difference) will produce different effects on activity of skeletal muscle enzymes, training of different intensity running (slope $2 \%$, speed 26, 30, 36 and $42 \mathrm{~m} / \mathrm{min}$ ), rat gastrocnemius muscle SDH activity appeared different changes, one of the most effective change in the $30 \mathrm{~m} / \mathrm{min}$ (equivalent to $83 \% \mathrm{VO} 2 \mathrm{max}$ ) groups, which may be related to the different training intensity on different skeletal muscle motor unit recruitment related [5]. In addition, living high training low increased the activity of SDH in skeletal muscle. It may also adapt to the reaction with HIF-1 and / or biological (such as promoting the increase anabolic hormone accelerate glycogen and protein synthesis).

Effect of hypoxia training during 4 weeks of rat skeletal muscle SDH activity is very significant. In a quiet state, normoxic control group and the other groups there were significant differences $(\mathrm{P}<0.01)$, intermittent hypoxic training group compared to normoxic control group increased by $56 \%$, compared with normoxia exercise group increased $43 \%$, explain rats after hypoxic training of 4 weeks later. The expression of SDH in rat skeletal muscle increased obviously.In the state immediately after exercise under normoxic control group and intermittent hypoxia training group, normoxic training group and intermittent hypoxia training group there were significant differences $(\mathrm{P}<0.01)$, and the normoxia control group and intermittent hypoxia control group, normoxic training group and intermittent hypoxia control group were significantly different $(\mathrm{P}<0.05)$, skeletal muscle SDH activity in intermittent hypoxia group, intermittent hypoxic training group increased by 58\% and 73\% respectively.After exercise state, normoxic control group and intermittent hypoxia control group, intermittent hypoxic exercise group, exercise group and intermittent hypoxia training group with a very significant difference $(\mathrm{P}<0.01)$, normoxic training group and intermittent hypoxia control group $(\mathrm{P}<0.05)$, the recovery period after exercise, whether hypoxia or hypoxic exercise, to improve the activity of $\mathrm{SDH}$ in rat skeletal muscle are obvious.According to the experimental results, the increase of three hours under the three conditions of gastrocnemius muscle SDH activity was quiet, immediately after, movement trend, and it can be seen in Figure 1, the three states, the activity of SDH in each group is a trend of increased first and then decreased, after exercise immediately reached a maximum value, then slowly return to the quiet state level. Hypoxia training of 4 weeks is likely to improve the suitable time of SDH enzyme activity in skeletal muscle of rats. 


\section{Conclusion}

Through 4 weeks of intermittent hypoxia training activity in rat skeletal muscle SDH improved significantly. The hypoxia plus exercise group improved obviously, that the organic combination of hypoxia and exercise is the best way to increase the activity of SDH in skeletal muscle of rats.

\section{References}

[1] Kolchinskaya,Tkatchouk EN. HypMedJ, 1993,(1):9-18.

[2] Shicheng Li,Ye Tian. Beijing Sport University, 1998,21 (2):15-181.

[3] Maoye Wang, Zhiping Lei. Of the Sixth National Sports Science Conference Abstracts, 2000: 205-2061.

[4] Qiang Li and so on. Of the Sixth National Sports Science Conference Abstracts, 2000: 207-208.

[5] Kaigang Li and so on. Chinese Journal of Sport Medicine, 2002, 21 (2): 166-1691. 